\title{
Gauß-Vorlesung in Regensburg mit Cédric Villani
}

\author{
Bernd Ammann, Thilo Küssner und Raphael Zentner
}

\begin{abstract}
Cédric Villani war der Hauptredner der Gauß-Vorlesung der DMV in Regensburg im Oktober 2017. Da Villani im Juni zuvor für die Partei En Marche! von Emmanuel Macron zum Abgeordneten des französischen Parlaments gewählt wurde, war es eine kleine Sensation, dass er den lange versprochenen Termin einhalten konnte. Obwohl die deutsche Presse die Veranstaltung nahezu ignorierte, war der wunderschöne Neuhaussaal des Regensburger Theaters fast bis auf den letzten Platz gefüllt und zur Vermeidung von Überfüllung mussten sogar kostenlose Platzkarten ausgegeben werden.
\end{abstract}

Die Gauß-Vorlesung der DMV findet zweimal im Jahr an wechselnden Orten statt. Ziel dieser Vorlesungen ist es, der mathematisch interessierten Öffentlichkeit einen Einblick in Ergebnisse der modernen Mathematik zu geben.

Die Gauß-Vorlesung für Herbst 2017 wurde am 23. Oktober vor ungefähr 360 Zuhörern im nahezu vollbesetzten Neuhaussaal des Regensburger Theaters gehalten. Höhepunkt der Veranstaltung war ein Vortrag von Cédric Villani zum Thema „Dreiecke, Gase, Preise und Menschen“, laut Villani die Geschichte der Begegnung von drei a priori nicht verwandten Gebieten: von nichteuklidischer Geometrie, von kinetischer Gastheorie und von Wirtschaftswissenschaften. Es ging aber auch um die Menschen, die an diesen Theorien gearbeitet haben und um diejenigen, die heute an ihnen weiterarbeiten. Hinter diesen Gebieten stehen sehr tiefgründige Konzepte: Ricci-Krümmung, Entropie eines Gases und optimaler Transport. Dennoch verstand es Villani, diese Ideen so zu erklären, dass auch ein Zuhörer mit wenigen Vorkenntnissen viel mit nach Hause nehmen konnte. Villani hat mit ähnlichen Vorträgen in Frankreich große Begeisterung hervorgerufen. Er verbindet somit die Exzellenz eines Fields-Medaillisten mit den Fähigkeiten eines großen Wissenschaftskommunikators.

Im ersten Teil der Gauß-Vorlesung begeisterte der Zauberer und gelernte Physiker Thomas Fraps das Publikum mit optischen Täuschungen, Seiltricks und einer Lösung des Zauberwürfels Rubik's Cube im Handumdrehen. Ebenfalls in die Gauß-Vorlesung integriert war die Verleihung des von Kaven-Preises an Manuel Amann aus Augsburg für Leistungen seiner Habilitation, die quaternionisch-Kähler-Mannigfaltigkeiten, Räume positiver Schnittkrümmung und rationale Homotopietheorie umfasst. In der Vorführung des Zauberers Fraps durften Manuel Amann und ein weiterer Gast in einem Kartenspiel gegen Fraps antreten. Ohne jeglichen Erfolg, wen wundert's! Ganz gegen die Traditionen der Zauberergilde, erklärte dann Fraps dem Publikum wie Gilbreaths Prinzip dazu führt, dass ein Zauberer das Spiel immer gewinnt.
Die Veranstaltung wurde von Bernd Ammann, Mathematiker an der Universität Regensburg, organisiert. Als er den Hauptsprecher der Gauß-Vorlesung, Cédric Villani, Anfang 2016 anfragte, konnte er nicht ahnen, dass Villani 2017 ein vielbeschäftigter Politiker sein würde. Villani trat im März 2017 als Direktkandidat der Partei En Marche! von Emmanuel Macron für das französische Parlament, die Assemblée Nationale, an und wurde im Juni mit großer Mehrheit als Direktkandidat gewählt. Inzwischen leitet er auch eine parlamentarische Kommission zur Förderung der künstlichen Intelligenz, wir berichten hierüber in diesem Heft (s. S. 67).

Umso erfreulicher war, dass Villani es dennoch geschafft hatte, seinen Besuch des Bundestags und der deutschen Regierung am Sonntag, den 22.10. und am Montagmorgen, den 23.10. mit einer intensiven Diskussion zu künstlicher Intelligenz in Regensburg und dann der Gauß-Vorlesung am Montagabend zu verbinden - schon am frühen Dienstagmorgen wartete auf ihn der nächste wichtige Termin in Paris.

Sehr erstaunlich war die geringe Resonanz der Presse, vor allem wenn man sie mit dem Echo vergleicht, das Villani in der französischen Presse und in der wissenschaftlich interessierten französischen Öffentlichkeit auslöst. In den wichtigsten Zeitungen und Zeitschriften in Frankreich ist Villani schon länger ein zentrales Thema, schon vor seiner Wahl ins Parlament und jetzt umso mehr. Als Beispiel: Am 17. Februar 2018 widmet sich das beigelegte Magazin der Tageszeitung Le Monde dem Thema "Cédric Villani, Parfait 'Premier de cordé' du Macronisme“, Villani als „Vorkletterer“ in der Seilschaft der Regierung Macron. Villanis Interview über künstliche Intelligenz ist die Titelgeschichte der berühmten Wochenzeitschrift L'Obs, (früher Le Nouvel Observateur) vom 7. März 2018.

Die Gauß-Vorlesung von Villani wurde in der deutschen Presse hingegen nicht einmal angekündigt, und trotz intensiver Bemühungen war die Berichterstattung auf einen kurzen und nicht gut recherchierten Artikel 


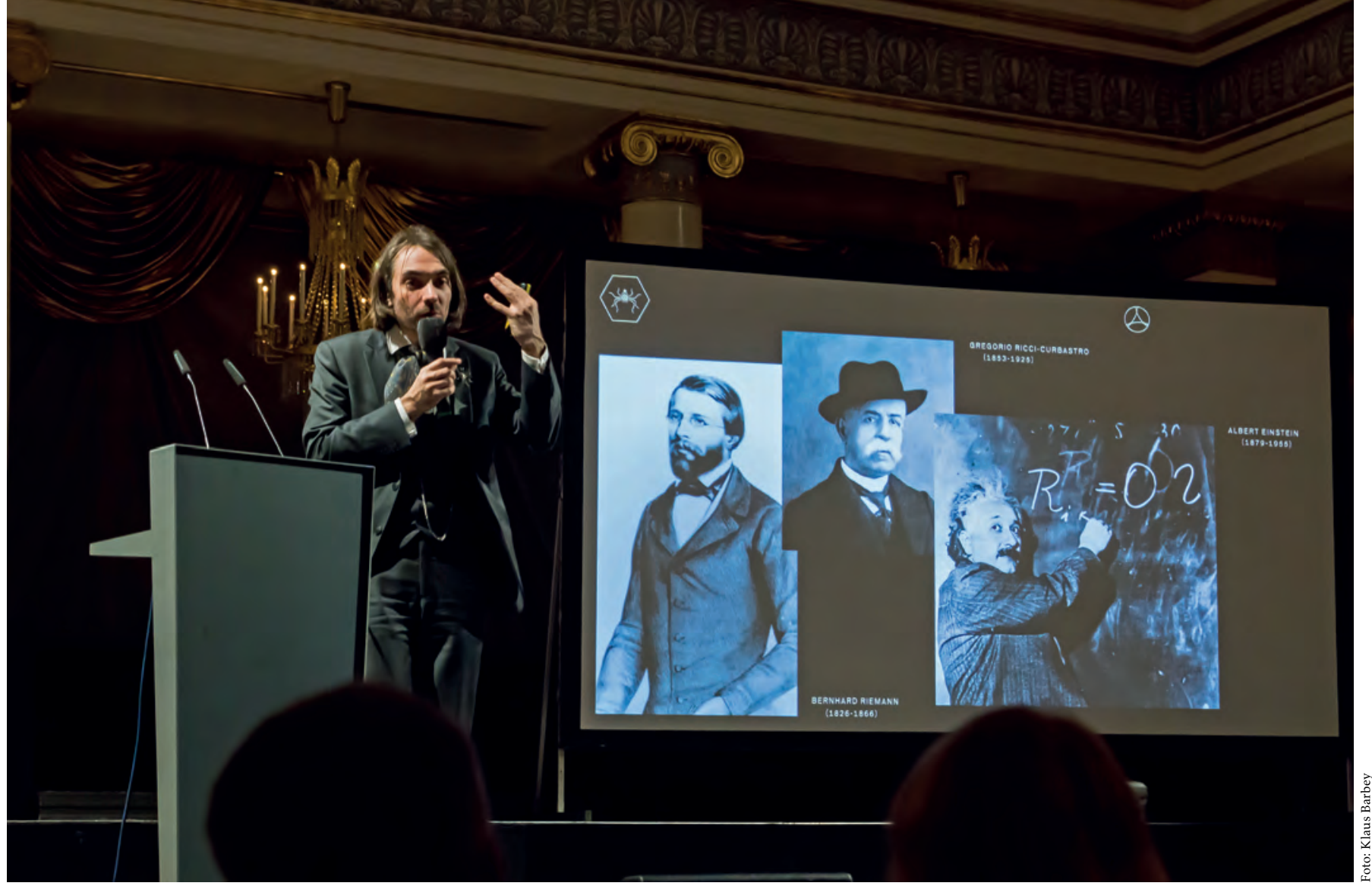

Gauß-Vorlesung in Regensburg: Cédric Villani erklärt Riemannsche Geometrie und ihre Anwendungen auf Allgemeine Relativitätstheorie

der Regensburger Lokalpresse reduziert. Natürlich ist Villani als französischer Mathematiker und Politiker in Frankreich viel präsenter, dennoch haben zum Beispiel seine Bemühungen zur Förderung künstlicher Intelligenz auch enorme Auswirkungen auf die deutsche Industrie und Gesellschaft, bis hin zum Koalitionsvertrag der aktuellen Großen Koalition. Der Organisator Bernd Ammann vermutet, dass die Gründe für die sehr unterschiedliche Resonanz insbesondere auf kulturelle Hintergründe zurückzuführen sind: in Frankreich die Sehnsucht nach einem Genie, von dem erwartet wird, dass es unter Nutzung des technischen Fortschritts die „Grande Nation“ zu neuen Erfolgen führt. Aktuelles Beispiel: Die Zeitung Le Monde und die Zeitschrift L'Obs werben derzeit für ihre neue Buchreihe „Génies des Mathématiques“. In Deutschland hingegen wird Fortschritt tendenziell kritischer gesehen, sicherlich oft aus guten Gründen, aber manchmal auch deswegen, weil wichtige mathematische und wissenschaftliche Tatsachen ignoriert werden.

Hinzu kommt auch, dass die Mathematik in Frankreich ein sehr hohes Renommée hat, was auch dazu führt, dass Frankreich im Bereich der mathematischen Forschung recht viele permanente Stellen finanziert, darunter viele CNRS-Stellen ohne Lehrverpflichtung. Die Zahl dieser Stellen ist nach wie vor hoch, obwohl in den letzten Jahren viele dieser Stellen leider gestrichen wurden. Zusätzlich gibt es in Frankreich eine viel ausgeprägtere Tradition, hochkarätige Mathematik populärwissenschaftlich an die Bevölkerung weiterzugeben. In Deutschland fehlt es hier nicht nur an personellen Kapazitäten an den Universitäten, sondern auch an engagierten und fachlich gebildeten Journalisten, mehr interessierten Zuhörern und einer größeren Verankerung solcher Aufgaben an unseren Universitäten.

Prof. Dr. Bernd Ammann und Dr. Raphael Zentner

Universität Regensburg, Fakultät für Mathematik, 9304o Regensburg bernd.ammann@mathematik.uni-regensburg.de raphael.zentner@mathematik.uni-regensburg.de

Dr. Thilo Kuessner,

Miltenbergstraße 8, 86199 Augsburg mathlog1@googlemail.com 